\title{
Remarques préliminaires sur le contexte éthique dans Soifs de Marie-Claire Blais
}

\author{
Irène Oore
}

Irene Oore reads Marie-Claire Blais' Soifs as a space of sustained inquiry, one in which the characters' thoughts are filled with questions and debate. In the pages of the novel, she finds settings akin to paradise and others that evoke unbearable human suffering. Such juxtaposed realities awaken the conscience of characters and readers alike, encouraging them to respond not with resignation, powerlessness, disregard or denial but with questions, with what Oore describes as ethical thinking. Blais' is an ethics of love, a practice of art and social action which counts its blessings rather than denouncing others with anger and exasperation: "Sometimes, it happens, there are angels on earth..." (Soifs)

Le dernier roman de Marie-Claire Blais, Soifs, ${ }^{1}$ publié en 1995, est une énorme mosaïque. Il serait impossible, à propos de ce roman, de parler d'une intrigue, il serait faux d'y voir une seule problématique autour d'un événement central. Soifs n'est bien évidemment ni un essai ni un traité philosophique, nous ne pouvons y chercher ni système éthique ni véritable théorie éthique, toutefois on peut considérer ce roman comme une espèce d'enquête soutenue à la fois ontologique et éthique, une investigation, un questionnement, ${ }^{2}$ tant au niveau des structures qu'au niveau thématique.

L'action entière (si l'on peut parler d'action) se déroule dans un décor paradisiaque, sur une île aux abords du golfe du Mexique. ${ }^{3}$ Cette île est un véritable microcosme d'une société, société où des êtres s'aiment et se déchirent, où les uns commettent des injustices et des crimes et les autres les subissent, où la maladie et la souffrance sont omniprésentes. Soifs présente donc, en les juxtaposant, le décor paradisiaque rêvé et la réalité humaine la plus brutale. Or, cette tension sous-jacente entre ce qui est (la réalité) et ce qui pourrait (et devrait) être (la valeur) constitue, nous le savons, la base même d'une réflexion éthique.

Le roman entier est une polyphonie de voix diverses, de monologues intérieurs qui s'interpellent, se font écho, parfois s'opposent l'un à l'autre, 
et se mettent en question. Souvent, d'ailleurs, il ne s'agit pas simplement d'une mise en question de l'autre, mais plutôt de la mise en question, plus difficile, plus essentielle aussi, de soi-même. Dans cette optique Soifs serait une investigation éthique de plusieurs consciences réfléchissant non seulement à leur parcours individuel, mais encore à la condition humaine en général. Car l'éthique examine les relations entre les individus ainsi que les relations des individus à eux-mêmes. L'éthique présuppose intériorisation et intériorité. La présentation de plusieurs voix évite le piège d'une voix autoritaire centrale et contribue à la problématisation et à la relativisation de certaines notions. Si nous parlons d'enquête et d'investigation, c'est que le texte pose (du moins de façon implicite) plusieurs questions telles que : «Comment être humain sur une île (et dans un monde) qui est une Sodome contemporaine ? ", "Où chercher la vérité, et par quels chemins essayer $\mathrm{d}^{\prime}$ y parvenir ? », « Ces limbes insulaires où se meuvent les personnages, seraient-ils en effet sans avenir (Sodome), ou au contraire constitueraientils la promesse d'une ère nouvelle possible (l'arche de Noé) ? $»^{4}$

Nous parlons aussi de recherche et de questionnement dans la mesure où le texte privilégie d'une façon frappante l'interrogatif. Presque chaque page du roman contient au moins une interrogation, et la plupart des pages en contiennent plusieurs. Ceci est clairement indicatif d'une façon de procéder. $^{5}$ Citons au hasard quelques-unes de ces propositions interrogatives : «si elle avait raison, n'avait-il pas commis quelque irréversible dommage » (Soifs 97), « le cas du chansonnier-tueur se livrant au carnage des jeunes filles, n'était-il pas un cas parmi tant d'autres » (143), et encore : " il se reprochait de n'avoir jamais su apprécier cette musique avant ce jour, dans quelle distraction avait-il vécu... » (175) ou « le temps n'était-il pas venu, pensait Charles, de se cloîtrer, de se soumettre à la règle du silence » (251). Les interrogations, indiquées par l'inversion du sujet et du verbe, souvent négatives, reviennent tout au long du roman dans ces monologues intérieurs, et agissent parfois tout simplement contre la crispation mentale et surtout contre la confiance de détenir la bonne réponse, parfois comme une question-souhait, une question-prière, une question-incantation aussi. Ces interrogations qui constituent une caractéristique essentielle des réflexions des personnages, indiquent, selon nous, une tension affective, un débat intérieur, un effort de modifier la conscience individuelle, et par là de modifier la conscience collective. ${ }^{6}$ C'est précisément en cela que ce mode d'être-en-question est le mode par excellence de la réflexion éthique. ${ }^{7}$ 
$* * *$

Si le Mal domine l'univers blaisien, on peut distinguer entre le Mal inévitable, celui de la maladie et de la mort, et le Mal que l'homme inflige à son prochain. C'est surtout ce dernier qui est l'objet du questionnement éthique. Il s'agit, entre autres, du racisme, du sexisme, du viol, de la violence, de la guerre ainsi que de l'injustice des lois. Face à ce Mal, certains ressentent de la honte, une honte résignée et impuissante, d'autres réagissent par la lassitude, le repliement sur soi (Soifs 276). Ainsi choisissent-ils de nier la réalité de la destruction nucléaire (189), dans leur lâcheté ils préfèrent oublier : « l'âme est chargée d'une éternité de peines, mais n'en continue pas moins de vivre dans l'oubli, le plaisir, l'insouciance » (16). Si l'oubli est le seul « paradis " possible, nous n'y avons guère droit. ${ }^{8}$ À travers les diverses voix, à travers les consciences variées, Soifs dresse une liste des horreurs que l'homme a fait et continue à faire subir à l'homme, pose la question de la responsabilité individuelle et collective pour les crimes passés et présents. De par cette énumération, Soifs s'oppose à l'oubli, rend impossible le déni. Des réflexions passagères, des inquiétudes profondes, voire de véritables hantises sont associées à un personnage donné (ou à un groupe de personnages) et sont justifiées par le passé et les circonstances présentes du personnage en question.

La peine de mort, sa cruauté, son irréversibilité hantent Renata. Le personnage nommé Mère est obsédé par les horreurs de la Deuxième Guerre mondiale et par l'Holocauste. Mélanie, sa fille, pense aux désastres nucléaires passés et à venir. Mama, qui s'inquiète pour ses enfants, note à plusieurs reprises l'ombre omniprésente du Ku Klux Klan. Si certaines préoccupations sont associées à un personnage, d'autres hantises sont partagées par plusieurs. C'est le cas, par exemple, des injustices associées à la condition féminine, à la guerre et à la pauvreté. D'ailleurs, souvent, le texte glisse imperceptiblement d'une conscience à l'autre et il semble difficile d'attribuer certaines réflexions à un personnage plutôt qu'à un autre. Soifs n'est donc, en quelque sorte, qu'une très longue énumération (souvent reprise et rappelant en ceci une litanie) des fléaux propres aux êtres humains. Au-delà de l'énumération se fait la description, et s'érige une véritable histoire de la violence. Les observations qui ont trait à ces crimes de l'humanité sont d'autant plus choquantes que les horreurs sont très fréquemment présentées en juxtaposition soit avec le décor paradisiaque de l'île, soit avec les festivités ininterrompues chez Mélanie et Daniel. La technique du contraste et de l'effet choc qui découle de la juxtaposition, 
domine le roman entier. Notons ainsi la dénonciation du militarisme par la description de l'intrusion du militaire dans le monde de la nature : « Et tout le long de l'océan, des bâtiments militaires alignés dans la périphérie de la plage » (21). La juxtaposition des touristes riches et repus qui viennent se soigner dans les îles, les stations balnéaires et thermales et les cadavres de la guerre du Golfe qui pourrissent dans le désert (38), scandalise. Les cris de joie des enfants en Amérique du Nord recevant leurs cadeaux de Hanukkah mis en parallèle avec les cris de détresse des enfants palestiniens à Gaza (291) révoltent.

Nous voyons donc que nommer le Mal, en révéler le scandale dans toute son ampleur, c'est déjà le dénoncer et le combattre. À ce niveau de dénonciation, l'écriture blaisienne est profondément engagée ${ }^{9}$ et constitue clairement un acte éthique : de par la fréquence des interrogations, de par les nombreuses juxtapositions des réalités dysphoriques, violentes et répréhensibles à des situations de joie, de célébration auxquelles plusieurs aspirent, Soifs provoque l'éveil de la conscience. Or la pratique d'une écriture éthique fait appel à une lecture également éthique et invite le lecteur à dévoiler la réalité individuelle et sociale, même si cette réalité est parfois répugnante et difficile à accepter.

\section{$* * *$}

Dans un article récent publié dans Quebec Studies et intitulé "Archeologies of an Uncertain Future in the Novels of Marie-Claire Blais ", ${ }^{10}$ Karen S. McPherson maintient que Blais propose très peu de réponses "réelles" aux horreurs qu'elle énumère. D'abord, il est essentiel, croyons-nous, de ne pas sous-estimer dans Soifs la valeur de la création de ce que nous nommons un espace-texte interrogatif. Alors que les assertions, les affirmations, bref, les « réponses » constituent un aboutissement et par là sont fermées aux multiples possibilités de la vie, alors que ces réponses résistent au changement, les interrogations n'ont guère la même valeur immuable, et s'ouvrent aux possibles infinis. Ce mode d'être dans l'interrogation est décrit ainsi par rapport à Mère : « Mère n'était-elle pas dépourvue de réponses devant le mystère de sa vie et de celle de ses enfants et petits-enfants, comme ces visages de beaux indigènes qui, dans les tableaux de Gauguin, se tournent vers le ciel rose de leur paradis en disant où allons-nous, sur cette terre » (152-53).

Si la prise de parole et l'énumération des maux constituent, comme nous l'avons dit, une dénonciation, le questionnement et l'interrogation 
constituent, nous l'avons indiqué, un effort en vue de transformer quelque chose en nous et par la suite dans la société. ${ }^{11}$ Dans un dialogue filmé Simone de Beauvoir disait : «Un roman, c'est une problématique. L'histoire de ma vie elle-même est une espèce de problématique et je n'ai pas à donner de solutions à des gens et les gens n'ont pas à attendre des solutions de moi ».12 Rester dans l'espace du questionnement et résister à la tent-ation d'avoir recours à des réponses rapides, est bien loin d'une solution de facilité, dans la mesure où ne pas savoir provoque une angoisse profonde. D'ailleurs, dans le contexte d'une réflexion éthique, et aussi paradoxal que cela puisse paraître, l'interrogation peut constituer parfois une réponse. Si le passé et ses crimes innombrables pèsent lourdement dans Soifs, le présent et l'avenir, bien que menacés, sont néanmoins ouverts. L'avenir de l'humanité sera-t-il celui de Sodome ou celui de $l^{\prime}$ Arche de Noé, se demande le lecteur tout au long du roman.

Plusieurs personnages blaisiens reconnaissent leur responsabilité morale et expriment le désir de se dépasser. Claude, le mari de Renata, réfléchit : « se dépasser, on ne rachetait jamais les fautes de ses pères, y auraitil enfin une génération d'hommes équitables, pensait-il » (15). Maintes tentatives du dépassement du Mal sont suggérées dans le texte. Ces tentatives, sans avoir l'arrogance d'une réponse satisfaite, néanmoins constituent des réponses, toujours et inévitablement mises en question. Les efforts de transcendance se situent dans Soifs à trois niveaux, le social, l'art et l'amour.

Mère imagine le désarmement (234) et croit que le combat contre le Mal se situe au niveau politique : «Mélanie devait combattre ces fléaux, encore quelques années... Mélanie pourrait fonder un parti politique car, c'était la vérité, Mélanie était une femme leader, elle en avait le caractère, comme le disait sa mère » (77). D'autres personnages voient le combat contre le Mal au niveau de la législation : "la législation des drogues réduirait les crimes, avait déclaré un juge à la retraite, tout ce qui était prohibé, tel l'alcool autrefois, contribuait à une vague de meurtres » (99). Le travail communautaire est une autre façon de combattre le Mal. Mélanie part en Afrique où elle s'engage dans une « lutte contre l'injustice et la pauvreté » (74) au niveau de la communauté, et le pasteur Jérémy ouvrira un mouroir dans l'île pour tous ceux qui « $\mathbf{n}^{\prime}$ ont pas de lieu pour rendre l'âme » (80).

À travers l'oeuvre blaisienne entière l'art combat le Mal. Ceci est aussi vrai dans Soifs. Renata croit que les jeunes qu'elle aime seront « capables de se dépasser, comme Franz, dans la musique » (14). Daniel et Mélanie 
rêvent à un paradis gouverné « par la liberté et la poésie » (235) et Ari, lui, croit que « l'art, la sculpture ... peuvent ... réhabiliter ... une partie de nous » (287) même si l'autre partie serait « dans un fossé boueux du Viêtnam » (287). Plusieurs personnages blaisiens, artistes, croient au triomphe ultime des arts : « l'Envahisseur, l'Occupant était à la porte mais l'écrivain avait été imperturbable dans son appartement de la rue de Fleurus, l'art, le langage, les lettres triompheraient de tout ... ils triompheraient de ces odieux et mesquins désordres des hommes, des débau-ches et des corruptions de leurs guerres " (224).

Dans la mesure où l'on peut parler d'une éthique dans l'oeuvre blaisienne entière, il faudrait parler d'une éthique de l'amour. L'amour, sous ses divers aspects combat le Mal. Dans Soifs c'est l'amour-charité du pasteur Jérémy, c'est aussi l'amour que ressent Mère pour Mélanie et que Mélanie à son tour ressent pour ses enfants à elle. C'est même l'amour physique chez les différents personnages. Nous sommes émus devant le dévouement, devant l'amour plein de compassion de Luc pour Jacques mourant du SIDA : " Luc dégageait Jacques de ses draps souillés, il le lavait, le nettoyait, tout en souriant et en bavardant, effaçait avec une serviette trempée dans l'eau de Cologne les traces de jus brunâtre sur les cuisses, le ventre de Jacques... Et Luc dit en prenant le malade dans ses bras, il faut dormir maintenant, je ne te quitterai plus » (56-57). Pour qu'un tel don de soi, une telle charité soient possibles, il faut évidemment que le personnage soit conscient d'autrui et de sa souffrance. Dans un autre passage, nous sommes témoins d'une empathie déchirante de "deux pompiers à genoux près d'un misérable brûlé qui vivait ses derniers instants ... si Dieu eût existé, eût-il pu agir avec plus de sympathie, d'empathie, que ces deux hommes impuissants devant la douleur d'autrui » (305-06). Enfin un personnage blaisien conclut: « il y a des anges, parfois, cela arrive, sur la terre ... partout, je le sais ... il y a des garçons et des filles comme toi ... l'univers est plein de cette sainteté méconnue des hommes, une sainteté profane et divine » (293). Cette « sainteté profane et divine » représente tout ce qui dans Soifs s'oppose au Mal.

$$
* * *
$$

Au coeur même du roman, nous trouvons quelques passages qui proposent une éthique étroitement liée à celle de l'amour, de l'art et même de l'action sociale, mais néanmoins distincte de celles-ci. C'est l'éthique de la reconnaissance et de la louange. C'est la mère de Mélanie qui évoque une 
sagesse venant de l'Est et de la tradition bouddhiste, mais une sagesse qui ne nous semble guère étrangère : plutôt que de dénoncer avec colère et exaspération, il s'agit de chanter sa gratitude pour les bienfaits reçus : «il y avait dans la gratitude, une harmonie, un équilibre certains » (178). Rappelons que l'effort éthique, qui répond à certaines aspirations de notre conscience, nous apporte précisément calme, équilibre et sérénité. Nous lisons encore par rapport à ce que nous nommons l'éthique de la reconnaissance : « la reconnaissance plutôt que la colère, plutôt que la déprécia-tion de soi-même, le chercheur japonais ne dénonçait-il pas les maux blasés de l'Occident, la culpabilité, la dépression » (179). En fin de compte, l'oeuvre blaisienne, comme toute oeuvre d'art, n'est-elle pas précisément cela, une expression de l'amour que l'on a pour le monde et un hymne à la vie?

\section{Notes}

${ }^{1}$ Marie-Claire Blais, Soifs, Montréal : Boréal, 1995. Toutes nos citations renvoient à l'édition ci-dessus.

${ }^{2}$ Notons le rapport intéressant entre le titre du roman, Soifs et l'interrogation. Soifs renvoient clairement dans le contexte blaisien au désir, et comporte une dimension affective évidente. C'est aussi le cas de la proposition interrogative. Nous lisons dans Le bon usage de Maurice Grévisse (Paris: Éditions Duculot, 1980) à la page 167: « Comme la proposition interrogative suppose généralement une certaine tension affective du sujet parlant, elle peut être rangée aussi parmi les propositions affectives ». Ce rapport entre la logique d'Éros et la logique érotétique (interrogative) est noté dans la présentation de Michel Meyer au numéro spécial de la revue Langue Française dédié à l'Interrogation, vol. 52 (déc 1981): 3-4. « logique érotétique - de erotesis qui signifie, en grec, interrogation et qui a en commun avec eros que ce dernier nous pousse à chercher ce qui nous manque, comme la question a besoin de trouver sa réponse ".

${ }^{3}$ S'agit-il de Key West où Marie-Claire Blais passe ses hivers ? S'agit-il de la Floride ? C'est possible, mais le nom de l'île n'est jamais mentionné, et nous ne croyons pas que la réponse à cette question soit fondamentale pour notre propos.

${ }^{4}$ Sodome et l'arche de Noé sont deux symboles récurrents dans Soifs.

${ }^{5}$ Les inversions indiquant l'interrogatif et des éléments lexicaux tels que « peut-être » et « il lui semble » abondent dans le roman. Ce sont tous 
des éléments qui fonctionnent comme modalisateurs d'assertion.

6 « C'est parce que je me demande quelle heure il est que je vous demande l'heure. Tout interrogatoire social est accompagné d'interrogations personnelles ». Leo Apostel, "De l'interrogation en tant qu'action », Langue Française 52 (déc 1981): 23.

${ }^{7}$ Notons que l'interrogation est bi-polaire, allant du pôle (+) au pôle (-) ou vice versa (voir Marc Wilmet, Grammaire critique du français, Louvain-laNeuve: Duculot, 1997, p. 452-53). D'autre part les valeurs qui sont au centre même de toute réflexion éthique ont, elles aussi, " un caractère de polarité. Leur hiérarchie est doublement orientée, en un sens positif et en un sans négatif. À chaque valeur s'oppose une contre-valeur: de même qu'au vrai s'oppose le faux et au beau le laid, au bien s'oppose le mal, et entre ces deux pôles, les valeurs s'étagent en gradation ascendante ou descendante " (Armand Cuvillier, Précis de philosophie, Paris: Librairie Armand Colin, 1966, p. 136).

${ }^{8}$ Lors de la remise du prix Nobel à Stockholm (10 décembre 1950) William Faulkner a dit: "Our tragedy today is a general and universal physical fear so long sustained by now that we can even bear it. There is only one question: When will I be blown up? Because of this, the young man or woman writing today has forgotten the problems of the human heart in conflict with itself $»$.

9 Voir à ce propos Jean-Paul Sartre, Qu'est-ce que la littérature? Paris: Gallimard, 1948.

${ }^{10}$ Karen S. McPherson, "Archetypes of an Uncertain Future in the Novels of Marie-Claire Blais », Quebec Studies 25 (1998): 93.

11 Voir à ce propos l'excellent article de Leo Apostel, « De l'interrogation en tant qu'action ", Langue Française 52 (déc 1981): 23-43.

12 Dayan Josée et Maka Ribowska, Simone de Beauvoir (film dialogue), Paris: Gallimard, 1979, p. 75. 